\title{
Silencing of active transposable elements in plants
}

In plant genomes the vast majority of transposable elements (TEs) are found in a transcriptionally silenced state that is epigenetically propagated from generation to generation. Although the mechanism of this maintenance of silencing has been well studied, it is now clear that the pathways responsible maintaining TEs in a silenced state differ from the pathways responsible for initially targeting the TE for silencing. Recently, attention in this field has focused on investigating the molecular mechanisms that initiate and establish TE silencing. Here we review the current models of how TEs are triggered for silencing, the data supporting each model, and the key future questions in this fast moving field.

\author{
Addresses \\ ${ }^{1}$ Department of Molecular Genetics, The Ohio State University, United \\ States \\ ${ }^{2}$ Center for RNA Biology, The Ohio State University, United States \\ Corresponding author: Slotkin, R Keith (Slotkin.2@osu.edu)
}

\section{Current Opinion in Plant Biology 2015, 27:xx-yy}

This review comes from a themed issue on Cell signalling and gene regulation

Edited by Xiaofeng Cao and Blake C Meyers

http://dx.doi.org/10.1016/j.pbi.2015.05.027

1369-5266/Published by Elsevier Ltd.

\section{Introduction}

Plant genomes are littered with TEs and their derived fragments, ranging from $15 \% \mathrm{TE}$ in the $135 \mathrm{MB}$ Arabidopsis thaliana genome to $85 \%$ of the TE-expanded $2500 \mathrm{MB}$ Zea mays genome. Although abundant, the vast majority of these TEs are not actively producing mRNAs or transposing, either due to their short fragmented nature or their transcriptionally-repressive silenced state. This highly efficient silencing of particularly the full-length autonomous TEs functions to neutralize the inherent mutagenic potential of TE activity produced from transposition events.

Although a great deal is known about how TE silencing is both maintained and reinforced (see [1-3] for more detail), very little is understood about how TEs arrived to this heterochromatic state in the first place. The exact duplicate nature of TE sequences demonstrates that TEs have invaded plant genomes and had boom periods of activity before the eventual bust of transcriptional silencing (for a recent example, see [4]). This Current Opinion focuses on recent research aimed at determining how an active TE is initially triggered for silencing, and how silencing marks are established and reinforced, resulting in the end-point state of maintenance of heterochromatic silencing.

\section{Initiation of silencing}

Two routes of initiation of silencing can occur, defined by whether or not existing small interfering RNAs (siRNAs) produced from previously silenced TE copies in the genome show sequence homology to the active copy.

\section{Homology-dependent initiation of silencing}

Plant genomes harbor tens of thousands of silenced TEs. These silenced TEs can be used to target active homologous TEs for RNA-directed DNA methylation (RdDM) and epigenetic silencing (see [5] for a review of RdDM). The well-studied RdDM pathway in plants can be split into upstream and downstream acting phases based on two RNA Polymerase II (Pol II) derivatives that target silenced TEs for non-protein coding transcription, called Pol IV and Pol V. In the upstream phase, Pol IV is recruited to previously silenced TEs through the transcriptionally repressive histone post-translational modification of histone $\mathrm{H} 3$ lysine 9 methylation (H3K9me) [6]. The TE transcript product of Pol IV is converted into double-stranded RNA by the protein RNA dependent RNA Polymerase 2 (RDR2) and subsequently cleaved into specifically sized 24-nucleotide (nt) siRNAs by Dicer-like 3 (DCL3) (Figure 1A). In all plants investigated, 24-nt siRNAs produced from silenced TEs are highly abundant [7-9]. In the downstream phase of the RdDM cycle, the 24-nt siRNAs are loaded into ARGONAUTE 4 (AGO4) and AGO6 and direct these proteins to nascent transcripts still associated with their chromatin template produced by Pol V. Pol V transcripts serve as a RNA scaffold on the chromatin that allows for siRNA-based silencing information to direct chromatin modification. Interaction between AGO4 or AGO6 and the Pol V transcript results in recruitment of a chromatin-modifying protein complex that directs $\mathrm{H} 3 \mathrm{~K} 9$ me and DNA methylation of the TE locus (Figure 1A) (see [10] for a review).

One function of these omnipresent 24-nt siRNAs is to act as a homology sensor, useful for identifying TEs with similar sequences to ensure that matching active TEs become silenced (Figure 1B). Multiple examples of the ability of the 24-nt siRNAs to silence active homologous TEs exist. For example in Arabidopsis, when TEs are activated or an Arabidopsis TE is transgenically placed 
Figure 1

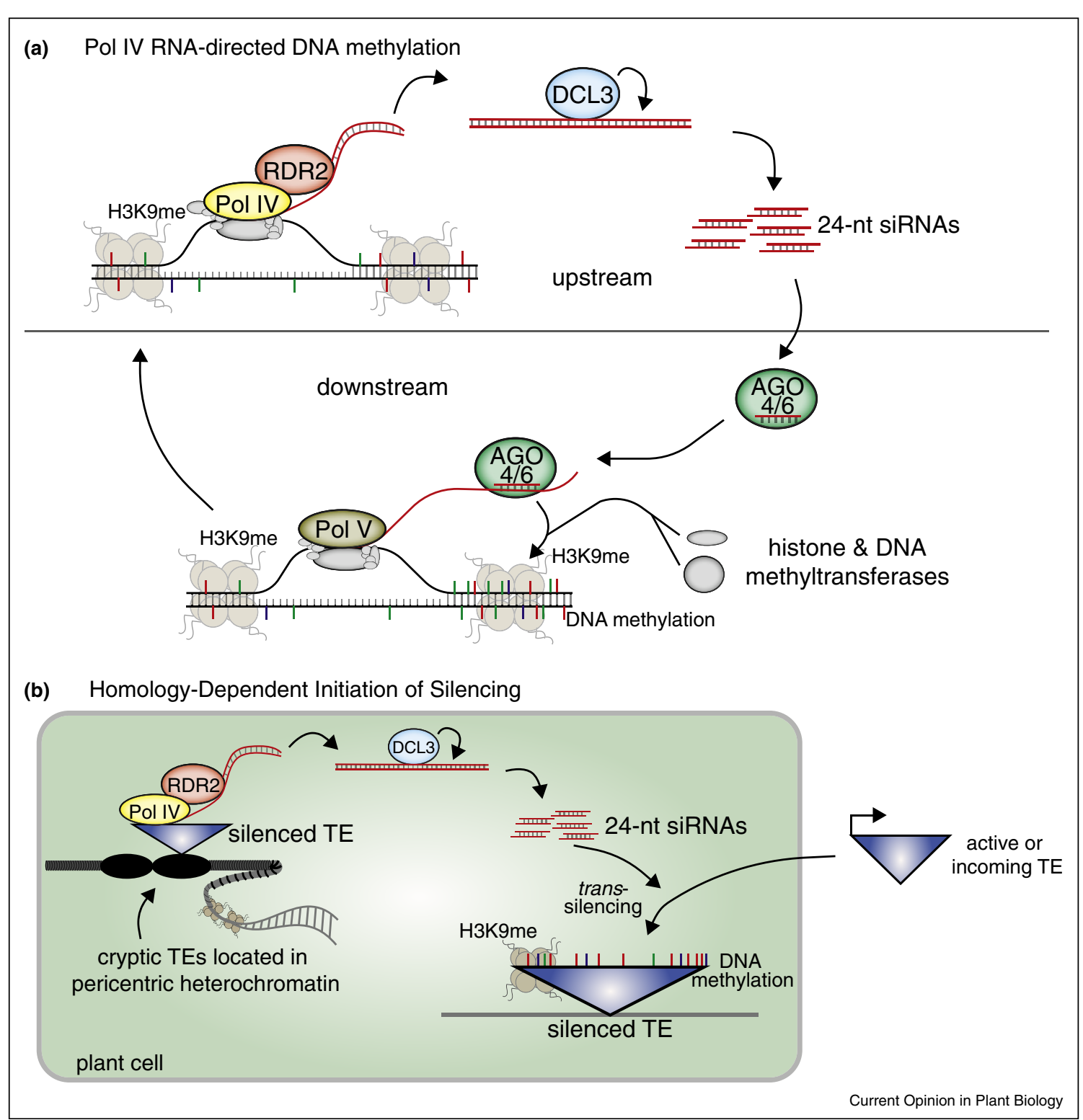

Pol IV RNA-directed DNA methylation and homology-dependent silencing. (A) The Pol II derivatives Pol IV and Pol V function in a RNA-directed DNA Methylation (RdDM) pathway to reinforce TE methylation through the activity of 24-nt siRNAs. In the upstream phase of this pathway Pol IV, RDR2 and DCL3 function to produce the 24-nt siRNAs from TEs associated with H3K9me. In the downstream phase of this pathway the interaction between a Pol V scaffolding transcript and a 24-nt siRNA-loaded AGO4 or AGO6 protein results in DNA methylation and H3K9me of the target TE. (B) Previously silenced and degenerated 'cryptic' TEs are abundant in plant genomes and are concentrated at the pericentromere. Pol IV transcribes these heterochromatic TEs, and the resulting non-coding RNAs are cleaved into 24-nt siRNAs by the activity of RDR2 and DCL3. The 24-nt siRNAs can function as a homology sensor to identify other TEs with similar sequences and initiate or reinforce their silencing by targeting $\mathrm{H} 3 \mathrm{~K} 9$ me and DNA methylation. DNA methylation is shown as red/CG, blue/CHG and green/CHH sticks, where $\mathrm{H}=\mathrm{A}, \mathrm{T}$ or $\mathrm{C}$.

back into the TE-silenced genome, the Pol IV/RDR2/ DCL3 (Pol IV-RdDM) components are necessary for the efficient re-silencing of these TEs $\left[11,12,13^{\bullet \bullet}\right]$. Likewise, in maize silencing of the Mutator TE family occurred when one TE copy underwent a rearrangement and created a long hairpin RNA producing abundant 24 to 25-nt siRNAs that resulted in the trans-silencing of active Mutator TEs at different loci [14]. Therefore, 24-nt Pol IV siRNAs produced from previously silenced or defective TE copies can act to trans-silence active copies (Figure 1B), and the silenced TEs within a plant genome provide the plant with an innate immunity against TE activity. An analogy has been made of the plant genome converting active TEs into cryptic (silenced and degenerated) or 'zombie' TEs, 
keeping them present in the genome for the use of silencing any active TEs of similar sequence [15]. Major questions in the Pol IV-RdDM pathway remain, such as how much homology is required between a 24-nt siRNA and its target transcript, and how Pol V is recruited to active TEs?

\section{Homology-independent initiation of silencing}

Recent research has shown a large number of TE horizontal transfers $\left[16^{\circ}, 17\right]$, which provide the TE the opportunity to colonize and proliferate in a new genome by circumventing the homology-based silencing pathway. New TE insertions may be silenced upon integration, as double-strand breaks are known to signal the recruitment of a small RNA-based silencing mechanism [18], however this has not been demonstrated to regulate TE activity. The common consensus is that when the homology-based silencing fails (due to lack of TE homology or failure of the Pol IV-RdDM pathway), before the TE is transcriptionally silenced, silencing must begin at the post-transcriptional level. Thus, TE mRNA degradation into 21 to 22-nt siRNAs via the plant's endogenous RNAi mechanism (which in Arabidopsis utilizes RDR6, AGO1, DCL2 and DCL4) [19,20] is the first trigger to eventual non-homology based TE silencing (Figure 2). In addition, RNAi represents a back-up system to post-transcriptionally silence TE activity when transcriptional silencing is lost, either during high stress, in Arabidopsis mutants, or developmental time points with TE transcriptional activity (see Box 1) [21-23]. The RNAi mechanism represents a self-reinforcing amplification loop that generates highly abundant 21 to 22-nt siRNAs from TE mRNAs (Figure 2). The products of RNAi, which are generated by RDR6, are secondary siRNAs able to work in trans and target other TE mRNAs with similar sequences, taking advantage of the repetitive nature of TEs to silence entire active TE families at once rather than individual elements. The double-stranded RNA products of RDR6 are cleaved by DCL4 and DCL2, which produce 21-nt and $22-n t$ siRNAs respectively $[24,25]$. The balance of 21 to 22-nt siRNAs is critical, as cleavage of a mRNA by a 22-nt siRNA is a known trigger for additional RDR6 activity and production of more secondary siRNAs [26,27]. The activity of DCL4 and production of 21-nt siRNAs, is an important decelerator to the system, preventing secondary siRNA production from spreading to similar gene sequences and degrading all mRNAs [28-30]. However, even without 22-nt siRNAs, targeting by multiple 21 -nt siRNAs has been shown in some cases to result in RDR6 activity and secondary siRNA production [31].

One major question is how RNAi is triggered to degrade TE mRNAs and not genic mRNAs. In non-plant species,

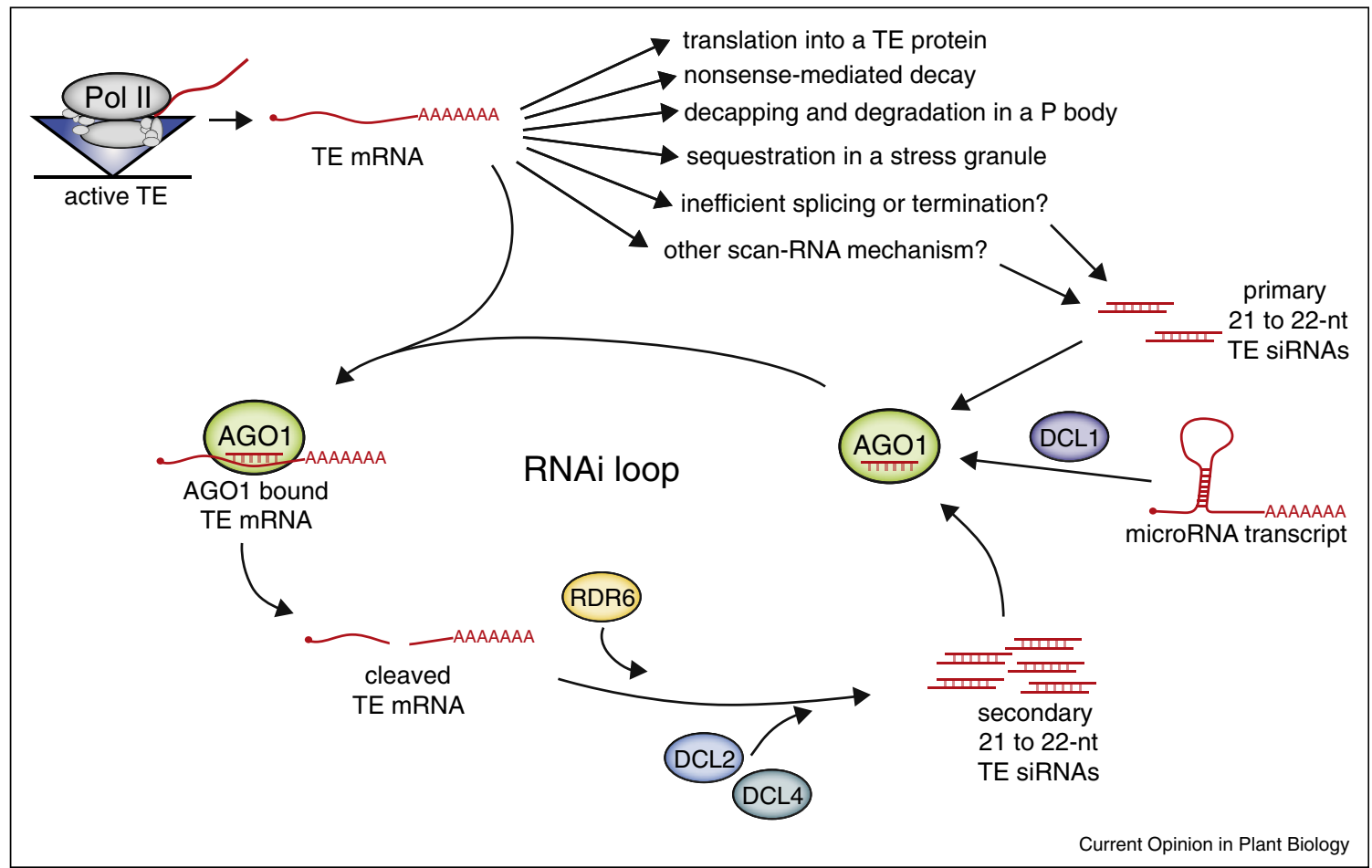

Multiple mechanisms trigger post-transcriptional silencing of TEs. TE mRNAs may be processed by a number of non-mutually exclusive pathways that lead to reduced translation. TE RNAi is initiated by microRNAs and potentially other mechanisms that result in the cleavage of TE mRNAs. Some cleaved TE mRNAs are then copied into double-stranded RNA by RDR6. DCL2 and DCL4 function to degrade TE double-stranded RNA and produce the abundant TE secondary siRNAs that direct additional rounds of RNAi, creating a loop of TE transcription and degradation. 
inefficient RNA splicing and incorrect termination trigger a transcript to be degraded by RNAi [32-35], and these may contribute to TE RNAi in plants (Figure 2). A major breakthrough in understanding the triggering of $\mathrm{TE}$ RNAi in plants came with the identification of TE mRNAs as microRNA targets. MicroRNAs are individual small RNAs specifically processed from stem-loop mRNA precursors that function to cleave and translationally repress complementary (usually genic) mRNA transcripts [36]. In a recent publication, known and new microRNAs were demonstrated to target the first cleavage event of some TE mRNAs (Figure 2) [37 $\left.7^{\bullet \bullet}\right]$. Although microRNAs are typically 21 -nt, some microRNAs are 22 -nt and trigger RDR6 production of double-stranded RNA from the targeted TE mRNA, initiating the cycle of RNAi (Figure 2). Although a conceptual breakthrough for identifying how TE mRNAs are selected for RNAi, how much this mechanism contributes to triggering RNAi compared to other potential mechanisms outlined in Figure 2 remains to be determined.

If a microRNA, secondary siRNA or other small RNA is required to initiate RNAi on a TE mRNA, this begs the question of where this complementary small RNA comes from in a case when a new TE enters a genome via horizontal transfer or transgenesis? One idea is that a TE mRNA can be triggered for RNAi without the requirement of an existing matching small RNA. A second idea that has gained popularity in non-plant organisms is that there are low level 'primal' or 'scan' small RNAs constantly produced that survey the transcriptome, randomly sampling mRNAs for cleavage and silencing. If a TE produces a mRNA substrate that is identified through targeting by a scanning small RNA, TE siRNAs can be generated and selectively enriched by RDRs (or the pingpong pathway for animal piRNAs) to generate the high level of repeat siRNAs required for TE silencing. In fission yeast AGO1 and in C. elegans 21U-RNAs perform cellular mRNA sampling and amplification of secondary siRNAs only occurs when antisense or foreign RNA substrate is available [38-40]. The cryptic microRNAs identified by $\left[37^{\circ}\right]$ that target TEs may represent this transcriptome surveillance mechanism in plants. Alternatively, a small amount of TE transcription may be allowed (even in TE-silenced cells), and due to the nested configuration of TEs these overlapping sense/antisense transcripts may be cleaved to produce a low level of small RNAs. These could then scan the transcriptome for sense TE mRNAs, initiating RNAi and post-transcriptional silencing of TE mRNA transcripts. Future experiments are required to determine if or how transcriptome scanning for TE mRNAs is accomplished in plants.

\section{Establishment of TE chromatin modification}

For transcriptionally active TEs that are subject to RNAi, the mechanism of how this post-transcriptional level silencing transitions to chromatin modification and the start of transcriptional silencing has been the focus of several recent publications. One model for this mechanism suggests that the first chromatin modification takes place only after a threshold copy number of elements is reached and enough mRNA is produced to overwhelm the DCL2 and DCL4 RNAi proteins, thus resorting to the DCL3 cleavage into 24-nt siRNAs (Figure 3) [41 ${ }^{\circ}$ ]. These 24-nt siRNAs then direct RdDM similar to their function when generated by Pol IV/RDR2. Therefore, this could represent a mechanism by which the plant could 'measure' the amount of aberrant transcript, choosing to switch to transcriptional silencing if the TE produces too much transcript and overwhelms RNAi. Although this mechanism is attractive, it does not account for loci that are highly expressed and targeted by RNAi that do not become methylated, nor regions of the genome that display transcript RNAi and RdDM without the presence of DCL3-dependent 24-nt siRNAs (such as gene-regulating trans-acting siRNA (tasiRNA) loci) [42].

A second mechanism has been recently reported whereby transcriptionally active TEs targeted by RNAi, and tasiRNA loci, are directly targeted for a form of RdDM that is not dependent on DCL3 or 24-nt siRNAs. In this mechanism, the 21 to 22-nt siRNAs that are directly produced from the RNAi of Pol II transcripts (by DCL2 and DCL4) can participate in a Pol II expression-dependent form of RdDM (Figure 3) $\left[13^{\circ *}, 42\right]$. Expression-dependent RdDM functions in both TE and virus establishment of silencing $\left[13^{\bullet \bullet}, 43\right]$. In the case of TE silencing, mutants were used that are defective in the maintenance of TE silencing, thus stalling and extending this establishment phase of silencing with the purpose of studying this phase of silencing with increased resolution. This work found that the 21 to 22-nt siRNAs are directly incorporated into the AGO6 protein, and similar to its role in Pol IV-RdDM (driven by 24-nt siRNAs), these 21 to 22-nt siRNAs direct AGO6 to chromatin targets using the Pol V scaffolding transcripts (Figure 3) $\left[44^{\circ}\right]$. This pathway was named RDR6-RdDM because of its dependence on the 21 to 22-nt siRNAs produced by RDR6, distinguishing it from the 24-nt driven Pol IV-RdDM $\left[13^{\bullet \bullet}\right]$. Why some 21 to 22 -nt siRNAs are incorporated and direct AGO6 and how Pol $\mathrm{V}$ is recruited to active TEs (providing specificity for TE targets of this pathway) are both currently unknown. In addition, this AGO6 mechanism may only represent one submechanism of the emerging field of (Pol II) expression-dependent DNA methylation [45]. For example, a protein called NERD was identified that participates in a hybrid pathway that utilizes both RNAi (RDR1, RDR6, DCL2, DCL4) and Pol IV-RdDM (Pol IV and DCL3) components to silence some noncoding RNA, newly arisen pseudogenes and a few TEs [46]. Although different specific pathways have been identified, the similarities between these non-mutually exclusive mechanisms are critically important and constitute a major breakthrough 


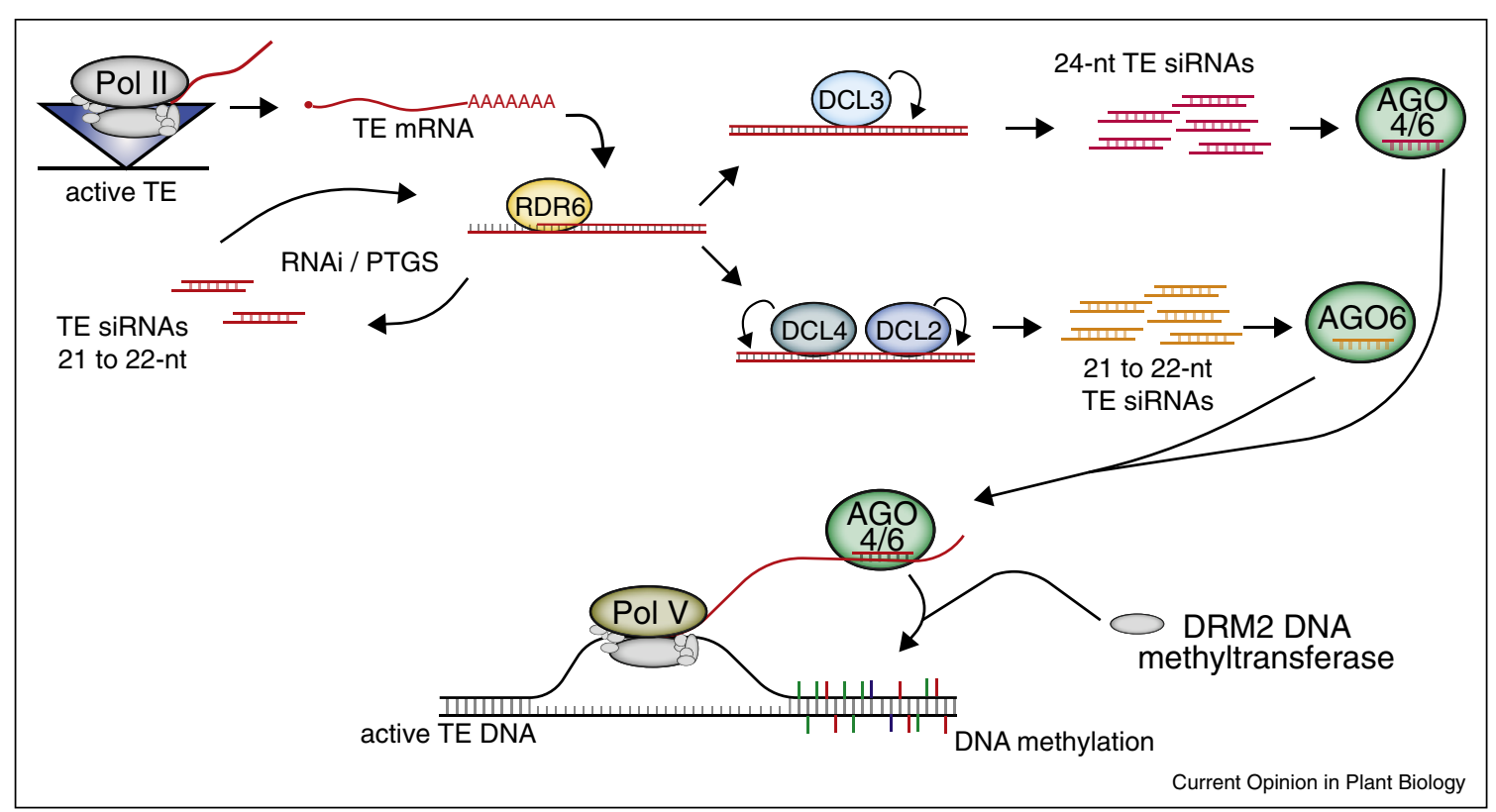

Establishment of RNA-directed DNA methylation. Once RNAi has been triggered, two models exist for how DNA methylation is established. These models differ in their dependence on DCL3 and which sized siRNA (21 to 22-nt vs 24-nt) targets the Pol V scaffolding transcript in the nucleus, establishing RNA-directed DNA methylation. PTGS = post-transcriptional gene silencing.

and advancement in this field: They define alternative RdDM mechanisms that are not solely dependent on Pol IV or RDR2, thus demonstrating that the establishment of TE chromatin marks can occur independently of the Pol IV-RdDM mechanism that is responsible for homology sensing and reinforcement of TE silencing.

\section{Reinforcement of silencing marks}

TE heterochromatic marks can be positively reinforced through the activity of the Pol IV-RdDM pathway and 24nt siRNAs (Figure 1) (see [5] for a recent review). Both Pol $\mathrm{IV}$ and Pol $\mathrm{V}$ are recruited to regions of the genome with previously established $\mathrm{H} 3 \mathrm{~K} 9 \mathrm{me}$ or DNA methylation $[6,47]$, and therefore this mechanism acts in two important ways. First, for regions that are associated with heterochromatic marks but not fully silenced, this mechanism acts as a self-reinforcing amplification loop to strengthen DNA and histone methylation patterns and keep TEs in a perpetually silenced state. Second, Pol IV-RdDM can spread the silencing marks to adjacent regions [48]. In Arabidopsis, which lacks the transcription-independent spreading of H3K9me by Heterochromatin Protein 1 [49,50], the Pol IV-RdDM pathway is probably the major mechanism responsible for the spread of heterochromatin. Both the reinforcement and spreading characteristics of Pol IVRdDM are particularly important for the transcriptional silencing of small TEs and TEs near genes. In the case of small TEs, there may not be enough nucleosome binding to maintain or 'hold' this TE region in a silenced state over time without the requirement for RdDM reinforcement. For TEs near genes, Pol IV-RdDM is required to define TE chromatin edges and sharp boundaries between heterochromatic TEs and euchromatic genes [51,52 $\left.{ }^{\bullet \bullet}\right]$. The Pol IV-RdDM pathway is therefore required to maintain transcriptional repression of a subset of small and euchromatic TEs in the Arabidopsis and maize genomes [53-55].

\section{Maintenance of TE silencing}

Similar to animals, plant TE DNA methylation and H3K9me (established by the RdDM pathways described above) can be copied and epigenetically transmitted from cell to cell across mitotic divisions without the requirement to be reset by additional RdDM. This epigenetic information is carried within the chromatin during cell division and can be propagated without the requirements of small RNAs. The protein MET1 acts to replicate patterns of symmetrical CG DNA methylation during cell division, while the proteins CMT2 and CMT3 act to maintain the methylation of $\mathrm{CHH}$ and $\mathrm{CHG}$ sites (where $\mathrm{H}=\mathrm{A}, \mathrm{T}$ or $\mathrm{C}$ ) guided by the H3K9me mark (Figure 4) [56,57]. These proteins thus ensure that each cell has the same DNA methylation as the progenitor cell. Some methylation, particularly in the CG context, can be efficiently copied at TEs to very high (>95\%) levels [58]. Many TEs in both the maize and Arabidopsis genomes are maintained in a transcriptionally silenced state only by the propagation of CG methylation and H3K9me, and do not require RdDM to reinforce this silencing $\left[44^{\bullet}, 58,59^{\bullet}, 60^{\circ}\right]$, although they 


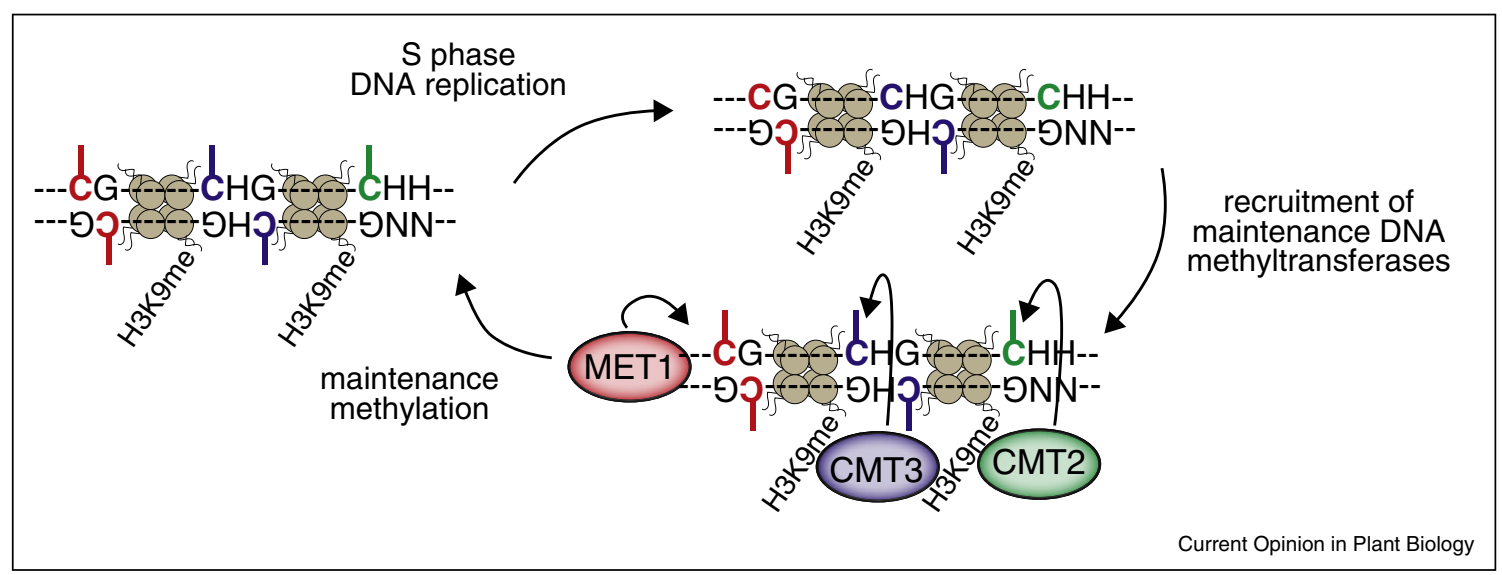

Maintenance of TE epigenetic silencing. TE DNA methylation in the CG sequence context (red) is propagated via the DNMT1 homolog MET1. TE CHG (blue) and $\mathrm{CHH}$ methylation (green) are maintained by CMT3 and CMT2, which are recruited to previously silenced TEs through their interaction with the heterochromatic mark $\mathrm{H} 3 \mathrm{~K} 9 \mathrm{me}$. In plants, TE DNA methylation is propagated from cell-to-cell, as well as from one generation to the next, resulting in trans-generational epigenetic inheritance of $T E$ silencing. $H=A, T$ or $C$.

will often produce Pol IV-dependent 24-nt siRNAs (see Homology-Dependent Initiation of Silencing section). In addition, a recent paper has identified a subset of TEs that may represent the evolutionarily transition from requiring reinforcement by Pol IV-RdDM to only requiring maintenance silencing. The authors termed these TEs 'double-locked' for their requirement of both Pol IV-RdDM and histone modification [61].

In plants, the propagation of chromatin information is not only passed across mitotic divisions, but is also maintained from one generation to another [62,63]. Although the trans-generational epigenetic silencing of TEs in plants is well established, in animals this topic remains controversial due to debate on the level of completeness of erasure of chromatin marks during animal reproduction and embryogenesis (reviewed in [64]). The lack of erasure of chromatin marks during development in plants means that the progress made towards silencing a TE where one generation left off can be picked-up and added to by the next generation (if the silencing occurred in the in the correct cell types, see Box 1). Thus, many of the RdDM mechanisms described above are not apparent on a generation-to-generation basis when TEs are maintained in a silenced state, but are only apparent when TEs reactivate or are transferred into a new genome. Once deeply silenced, TE sequences will begin to accumulate mutations due to the lack of selective pressure for their activity, and the TE will degrade into a cryptic or unrecognizable sequence.

\section{Remaining active despite multiple mechanisms of repression}

In wild-type cells with functional homology-dependent and -independent silencing mechanisms, occasionally some TE copies are able to remain active. For example, the ONSEN retrotransposon acquired a heat-responsive regulatory element that leads to $\mathrm{TE}$ activation upon heat stress despite functional silencing mechanisms [12,23]. Another example is the Arabidopsis Evade'TE, which was discovered because once activated it escapes corrective reestablishment of silencing and transposes into genes, even though there are closely related silenced family members present in the genome [76]. The ability of Evadéto avoid silencing is probably due to the lack of TE siRNAs that it or its closely related family members produce until transpositions result in a higher copy number. Additionally the encapsulation of its mRNA into gag protein particles sequesters it from 21 to $22-n t$ siRNAs $\left[41^{\bullet}\right]$. In other cases, the lack of silencing is puzzling because silenced members of the same TE family within the same genome are producing abundant Pol IV-dependent 24-nt siRNAs that should trans-silence the active copy. For example, in maize individual Mutator family TE copies are able to remain active despite abundant 24-nt siRNAs generated from silenced and fragmented homologous copies [77]. The activity of these single elements could potentially result in a period of high activity for the TE family. These booms in amplification occasionally (on evolutionary timescales) occur, leaving their relics and signatures of recent activity in plant genomes [78]. Therefore, a major question in the field is how can individual elements remain active in the same genome with other family members that are silenced?

Multiple mechanisms of TE evasion of silencing have been proposed, such as by the TE encoding a suppressor of gene silencing or by the TE becoming an adaptive benefit to the regulation of a critical gene [79-81]. 


\section{Box 1 Role of Development in TE Silencing}

Although this Current Opinion article focuses on the mechanisms and pathways responsible for TE silencing, all of these pathways are not functional in all tissues or developmental time points. Plant development exerts a spatial and temporal level of regulation on TEs. For example, the RDR6-RdDM pathway outlined in Figure 3 is dependent on the function of AGO6, which is only expressed in meristematic tissues [44], while reinforcement by Pol IV-RdDM and maintenance of DNA methylation occur more broadly across different organ and tissue types. Data has demonstrated that the shoot apical meristem and floral meristem, which produce the germ cells, are critical for the proper silencing of TEs [65], thus ensuring that the initiation or establishment of TE silencing accomplished in one generation is epigenetically passed to the next generation.

In addition to the developmental-specific timing of the silencing of TEs, there are particular points in normal plant development that undergo developmental relaxation of TE silencing (DRTS) [66]. In the wild-type pollen grain, the pollen vegetative nucleus undergoes a loss of heterochromatin and global reactivation of TE expression, followed by transposition $[67,68]$. In the pollen vegetative nucleus, TEs undergo the $\mathrm{CHH}$ hypermethylation associated with transcriptional activity and expression-dependent RdDM [69]. In addition, the analogous central cell in the female gametophyte shows signatures of chromatin decondensation [70], and after fertilization the endosperm displays DRTS [71,72]. Both the pollen vegetative nucleus and endosperm are terminally differentiated tissues, and it has been proposed that the DRTS events play a role in providing information content about TEs (small RNAs or other) to the germinal sperm cells or embryo [68,71]. It has been suggested that the DRTS events are preparing the critical germ cells for a confrontation with potentially active TEs upon cross fertilization, which will then be consolidated into epigenetically silenced heterochromatin [73], similar to the TE silencing/hybrid dysgenesis mechanism found in Drosophila [74]. Similarly, a DRTS event in maize leaves at the time of transition from juvenile to adult vegetative growth has been suggested to provide TE information back to the meristem to efficiently silence TEs where it matters most: first, for the production of future haploid gametophytes with only one copy of each gene and second, for the next generation [75]. However, in all of these cases there is no clear understanding if the DRTS events result in a lasting or heritable reinforcement or triggering of TE silencing. Critical questions to define the functional role of DRTS events (if any) remain unanswered.

Although examples of many of these mechanisms exist $[82,83]$, the mechanism that has gained the most attention is TE integration into an active gene, thus hiding and/or preventing the TE from silencing. In the case of the Mutator TEs mentioned above, the position of the element and proximity to a gene is critical for evasion of silencing [84]. In Arabidopsis a systematic cataloging of active Mutator TEs identified insertion sites in genic $3^{\prime}$ untranslated regions as safe havens to avoid silencing [85]. The authors proposed that the TE copies were insulated from silencing due to the selective pressure to maintain the gene in an active chromatin configuration. Because different TE families have different insertion site preferences, the ability to circumvent TE silencing may be higher for some DNA TEs (such as Mutator) that target genic regions for insertion, and lower for LTR retrotransposons that typically target heterochromatin.
A major question that remains is whether a given TE copy inserted near a gene can avoid silencing altogether, or whether silencing is targeted but then reversed? For example, the TE may be completely resistant to de novo chromatin modification, potentially due to the downstream Pol V chromatin-modifying complex not being targeted to this genic region. This could account for the production of Pol IV-dependent 24-nt siRNAs by the silenced TE family members, but their lack of activity on the genic TE insertion. Alternatively, Pol IV-RdDM may target a genic TE copy for silencing, but subsequently this silencing is over-written by DNA and histone demethylases to maintain a euchromatic environment around the gene. In Arabidopsis, the histone demethylase IBM1 and DNA glycosylases (which remove methylated DNA) ROS1, DME, DML2 and DML3 are required to keep genic sequences near TE insertion sites in the proper euchromatic environment [86-88]. It will be critical to understand if and how TE copies can avoid silencing, or what regulates the demethylation machinery to overwrite TE silencing, as the answer to this question will provide insight on how to manipulate TE, transgene and endogenous gene expression.

\section{Conclusion}

Three years ago the known mechanisms responsible for regulating TEs were confined to Pol IV-RdDM and the maintenance of DNA methylation. More recently, multiple independent groups have identified diverse mechanisms that the plant uses to repress transcriptionally active TEs, adding a new level of detail and function to post-transcriptional silencing pathways. Although examples of multiple molecular mechanisms responsible for silencing TEs are described here, how these individual mechanisms are ordered and link together is currently unknown. Models have been generated that attempt to assemble these pathways in a linear fashion $[5,43,89]$, but currently these pathways exist as individual modules that may fit together in a number of different arrangements. Future research is required to assemble the progression of silencing of an active TE.

\section{Acknowledgements}

D.F. is supported by the U.S. National Institutes of Health training grant T32 GM086252. The Slotkin lab is supported by U.S. National Science Foundation grant MCB-1252370.

\section{References and recommended reading}

Papers of particular interest, published within the period of review, have been highlighted as:

- of special interest

$\bullet$ of outstanding interest

1. Lisch D: Epigenetic regulation of transposable elements in plants. Ann Rev Plant Biol 2009, 60:43-66.

2. Bucher E, Reinders J, Mirouze M: Epigenetic control of transposon transcription and mobility in Arabidopsis. Curr Opin Plant Biol 2012, 15:503-510. 
3. Ito H, Kakutani T: Control of transposable elements in Arabidopsis thaliana. Chromosome Res 2014, 22:217-223.

4. Willing E-M, Rawat V, Mandáková T, Maumus F, James GV, Nordström KJV, Becker C, Warthmann N, Chica C, Szarzynska B et al.: Genome expansion of Arabis alpina linked with retrotransposition and reduced symmetric DNA methylation. Nat Plants 2015, 1:14023.

5. Matzke MA, Mosher RA: RNA-directed DNA methylation: an epigenetic pathway of increasing complexity. Nat Rev Genet 2014, 15:394-408

6. Law JA, Du J, Hale CJ, Feng S, Krajewski K, Palanca AMS, Strahl BD, Patel DJ, Jacobsen SE: Polymerase IV occupancy at RNA-directed DNA methylation sites requires SHH1. Nature 2013, 498:385-389.

7. Nobuta K, Venu RC, Lu C, Beló A, Vemaraju K, Kulkarni K, Wang W, Pillay M, Green PJ, Wang G-L et al.: An expression atlas of rice mRNAs and small RNAs. Nat Biotechnol 2007, 25:473-477.

8. Cho SH, Addo-Quaye C, Coruh C, Arif MA, Ma Z, Frank W, Axtell MJ: Physcomitrella patens DCL3 is required for 22-24 nt siRNA accumulation, suppression of retrotransposon-derived transcripts, and normal development. PLOS Genet 2008, 4:e1000314.

9. Huang Y, Kendall T, Mosher RA: Pol IV-dependent siRNA production is reduced in Brassica rapa. Biology (Basel) 2013, 2:1210-1223.

10. Wierzbicki AT: The role of long non-coding RNA in transcriptional gene silencing. Curr Opin Plant Biol 2012, 15:517-522.

11. Teixeira FK, Heredia F, Sarazin A, Roudier F, Boccara M, Ciaudo C Cruaud C, Poulain J, Berdasco M, Fraga MF et al.: A role for RNAi in the selective correction of DNA methylation defects. Science 2009, 323:1600-1604.

12. Ito $\mathrm{H}$, Gaubert $\mathrm{H}$, Bucher E, Mirouze M, Vaillant I, Paszkowski J: An siRNA pathway prevents transgenerational retrotransposition in plants subjected to stress. Nature 2011, 472:115-119.

13. Nuthikattu S, McCue AD, Panda K, Fultz D, DeFraia C, Thomas EN,

- Slotkin RK: The initiation of epigenetic silencing of active transposable elements is triggered by RDR6 and 21-22 NUCLEOTIDE SMALL INTERFERing RNAs. Plant Physiol 2013, 162:116-131

This publication showed that in addition to the methylation that the Pol IV/ $24 n t$ siRNA pathway targets to silenced TEs, 21-22nt siRNAs produced from transcriptionally active TE mRNAs can also target DNA methylation.

14. Slotkin RK, Freeling M, Lisch D: Heritable transposon silencing initiated by a naturally occurring transposon inverted duplication. Nat Genet 2005, 37:641-644.

15. Lisch D, Bennetzen JL: Transposable element origins of epigenetic gene regulation. Curr Opin Plant Biol 2011, 14: 156-161.

16. El-Baidouri M, Carpentier M-C, Cooke R, Gao D, Lasserre E,

- Llauro C, Mirouze M, Picault N, Jackson SA, Panaud O: Widespread and frequent horizontal transfers of transposable elements in plants. Genome Res 2014, 24:831-838.

This publication detailed that over half of plant genomes sequenced to date show strong evidence of TE horizontal transfer, demonstrating that TEs are continuously invading new genomes.

17. Diao $\mathrm{X}$, Freeling M, Lisch D: Horizontal transfer of a plant transposon. PLOS Biol 2006, 4:e5.

18. Wei W, Ba Z, Gao M, Wu Y, Ma Y, Amiard S, White Cl, Rendtlew Danielsen JM, Yang Y-G, Qi Y: A role for small RNAs in DNA double-strand break repair. Cell 2012, 149:101-112.

19. Dunoyer $P$, Brosnan $C A$, Schott $G$, Wang $Y$, Jay F, Alioua A, Himber C, Voinnet O: An endogenous, systemic RNAi pathway in plants. EMBO J 2010, 29:1699-1712.

20. McCue AD, Nuthikattu S, Reeder SH, Slotkin RK: Gene expression and stress response mediated by the epigenetic regulation of a transposable element small RNA. PLOS Genet 2012, 8:e1002474.
21. Dowen RH, Pelizzola M, Schmitz RJ, Lister R, Dowen JM, Nery JR Dixon JE, Ecker JR: Widespread dynamic DNA methylation in response to biotic stress. Proc Natl Acad Sci U S A 2012, 109:E2183-E2191.

22. Sarkies P, Selkirk ME, Jones JT, Blok V, Boothby T, Goldstein B Hanelt B, Ardila-Garcia A, Fast NM, Schiffer PM et al.: Ancient and novel small RNA pathways compensate for the loss of piRNAs in multiple independent nematode lineages. PLOS Biol 2015, 13:e1002061.

23. Cavrak VV, Lettner N, Jamge S, Kosarewicz A, Bayer LM, Scheid OM: How a retrotransposon exploits the plant's heat stress response for its activation. PLoS Genet 2014 10:e1004115.

24. Xie Z, Allen E, Wilken A, Carrington JC: DICER-LIKE 4 functions in trans-acting small interfering RNA biogenesis and vegetative phase change in Arabidopsis thaliana. Proc Natl Acad Sci U S A 2005, 102:12984-12989.

25. Deleris A, Gallego-Bartolome J, Bao J, Kasschau KD, Carrington JC, Voinnet O: Hierarchical action and inhibition of plant Dicer-like proteins in antiviral defense. Science 2006 , 313:68-71.

26. Cuperus JT, Carbonell A, Fahlgren N, Garcia-Ruiz H, Burke RT, Takeda A, Sullivan CM, Gilbert SD, Montgomery TA, Carrington JC: Unique functionality of 22-nt miRNAs in triggering RDR6-dependent siRNA biogenesis from target transcripts in Arabidopsis. Nat Struct Mol Biol 2010, 17:997-1003.

27. Chen H-M, Chen L-T, Patel K, Li Y-H, Baulcombe DC: Wu S-H: $22-$ Nucleotide RNAs trigger secondary siRNA biogenesis in plants. Proc Natl Acad Sci U S A 2010, 107:15269-15274.

28. Mlotshwa S, Pruss GJ, Peragine A, Endres MW, Li J, Chen X, Poethig RS, Bowman LH, Vance V: DICER-LIKE2 plays a primary role in transitive silencing of transgenes in Arabidopsis. PLOS ONE 2008, 3:e1755.

29. Wang X-B, Jovel J, Udomporn $P$, Wang $Y$, Wu Q, Li W-X, Gasciolli V, Vaucheret H, Ding SW: The 21-nucleotide, but not 22-nucleotide, viral secondary small interfering RNAs direct potent antiviral defense by two cooperative argonautes in Arabidopsis thaliana. Plant Cell 2011, 23 : 1625-1638.

30. Zhang X, Zhu Y, Liu X, Hong X, Xu Y, Zhu P, Shen Y, Wu H, Ji Y, Wen $X$ et al: : Suppression of endogenous gene silencing by bidirectional cytoplasmic RNA decay in Arabidopsis. Science 2015, 348:120-123.

31. Axtell MJ, Jan C, Rajagopalan R, Bartel DP: A two-hit trigger for siRNA biogenesis in plants. Cell 2006, 127:565-577.

32. Pane $A$, Jiang $P$, Zhao DY, Singh $M$, Schüpbach $T$ : The Cutoff protein regulates piRNA cluster expression and piRNA production in the Drosophila germline. EMBO J 2011, 30:4601-4615.

33. Dumesic PA, Natarajan P, Chen C, Drinnenberg IA, Schiller BJ, Thompson J, Moresco JJ, Yates JR, Bartel DP, Madhani HD: Stalled spliceosomes are a signal for RNAi-mediated genome defense. Cell 2013, 152:957-968.

34. Goriaux C, Desset S, Renaud Y, Vaury C, Brasset E: Transcriptional properties and splicing of the flamenco piRNA cluster. EMBO Rep 2014, 15:411-418.

35. Malone CD, Mestdagh C, Akhtar J, Kreim N, Deinhard P, Sachidanandam R, Treisman J, Roignant J-Y: The exon junction complex controls transposable element activity by ensuring faithful splicing of the piwi transcript. Genes Dev 2014, 28:1786-1799.

36. Meyers BC, Axtell MJ, Bartel B, Bartel DP, Baulcombe D, Bowman JL, Cao X, Carrington JC, Chen X, Green PJ et al.: Criteria for annotation of plant MicroRNAs. Plant Cell 2008, 20:3186-3190.

37. Creasey KM, Zhai J, Borges F, Van Ex F, Regulski M, Meyers BC

-• Martienssen RA: miRNAs trigger widespread epigenetically activated siRNAs from transposons in Arabidopsis. Nature 2014, 508:411-415. 
This publication provided evidence that TE mRNAs are widely targeted by known and newly identified microRNAs, post-transcriptionally repressing TE activity and initiating TE RNAi.

38. Halic M, Moazed D: Dicer-independent primal RNAs trigger RNAi and heterochromatin formation. Cell 2010, 140:504-516.

39. Lee H-C, Gu W, Shirayama M, Youngman E, Conte D, Mello CC: Celegans piRNAs mediate the genome-wide surveillance of germline transcripts. Cell 2012, 150:78-87.

40. Shirayama M, Seth M, Lee H-C, Gu W, Ishidate T, Conte D, Mello CC: piRNAs initiate an epigenetic memory of nonself RNA in the C. elegans germline. Cell 2012, 150:65-77.

41. Marí-Ordóñez A, Marchais A, Etcheverry M, Martin A, Colot V,

-. Voinnet O: Reconstructing de novo silencing of an active plant retrotransposon. Nat Genet 2013, 45:1029-1039.

This publication observed the resilencing of an Arabidopsis TE over multiple generations. The TE was initially protected from RNAi by its gag retrotransposon capsid, and then eventually triggered for DNA methylation and transcriptionally silenced.

42. Wu L, Mao L, Qi Y: Roles of DICER-LIKE and ARGONAUTE proteins in TAS-derived small interfering RNA-triggered DNA methylation. Plant Physiol 2012, 160:990-999.

43. Bond DM, Baulcombe DC: Epigenetic transitions leading to heritable RNA-mediated de novo silencing in Arabidopsis thaliana. Proc Natl Acad Sci U S A 2015, 112:917-922.

44. McCue AD, Panda K, Nuthikattu S, Choudury SG, Thomas EN,

- Slotkin RK: ARGONAUTE 6 bridges transposable element mRNA-derived siRNAs to the establishment of DNA methylation. EMBO J 2015, 34:20-35

This publication demonstrated that 21-22nt siRNAs derived from TE mRNAs can direct an Argonaute protein to its transcriptionally active TE targets, transitioning from post-transcriptional silencing to chromatin modification.

45. Sasaki T, Lee T-F, Liao W-W, Naumann U, Liao J-L, Eun C, Huang Y-Y, Fu JL, Chen P-Y, Meyers BC et al.: Distinct and concurrent pathways of Pol II- and Pol IV-dependent siRNA biogenesis at a repetitive trans-silencer locus in Arabidopsis thaliana. Plant J 2014, 79:127-138.

46. Pontier D, Picart C, Roudier F, Garcia D, Lahmy S, Azevedo J, Alart E, Laudié M, Karlowski WM, Cooke R et al:: NERD, a plantspecific GW protein, defines an additional RNAi-dependent chromatin-based pathway in Arabidopsis. Mol Cell 2012, 48:121-132.

47. Johnson LM, Du J, Hale CJ, Bischof S, Feng S, Chodavarapu RK Zhong X, Marson G, Pellegrini M, Segal DJ et al.: SRA- and SETdomain-containing proteins link RNA polymerase $V$ occupancy to DNA methylation. Nature 2014, 507:124-128.

48. Daxinger L, Kanno T, Bucher E, van der Winden J, Naumann U, Matzke AJM, Matzke M: A stepwise pathway for biogenesis of 24-nt secondary siRNAs and spreading of DNA methylation. EMBO J 2009, 28:48-57.

49. Zhang X, Germann S, Blus BJ, Khorasanizadeh S, Gaudin V, Jacobsen SE: The Arabidopsis LHP1 protein colocalizes with histone H3 Lys27 trimethylation. Nat Struct Mol Biol 2007, 14:869-871.

50. Turck F, Roudier F, Farrona S, Martin-Magniette M-L, Guillaume E, Buisine N, Gagnot S, Martienssen RA, Coupland G, Colot V: Arabidopsis TFL2/LHP1 specifically associates with genes marked by trimethylation of histone H3 lysine 27. PLOS Genet 2007, 3:e86.

51. Zhong X, Hale CJ, Law JA, Johnson LM, Feng S, Tu A, Jacobsen SE: DDR complex facilitates global association of RNA polymerase $\mathbf{V}$ to promoters and evolutionarily young transposons. Nat Struct Mol Biol 2012, 19:870-875.

52. Zemach A, Kim MY, Hsieh P-H, Coleman-Derr D, Eshed-Williams L,

- Thao K, Harmer SL, Zilberman D: The Arabidopsis nucleosome remodeler DDM1 allows DNA methyltransferases to access $\mathrm{H} 1$ containing heterochromatin. Cell 2013, 153:193-205.

This publication was the first to separate the regulation of silenced TEs based on maintenance methylation factors DDM1 and linker histone $\mathrm{H} 1$ from the TEs regulated by RdDM. They showed that upon transcriptional reactivation, TEs not previously targeted now undergo RdDM.
53. Onodera Y, Haag JR, Ream T, Costa-Nunes P, Pontes O, Pikaard CS: Plant nuclear RNA polymerase IV mediates siRNA and DNA methylation-dependent heterochromatin formation. Cell 2005, 120:613-622.

54. Huettel B, Kanno T, Daxinger L, Aufsatz W, Matzke AJM, Matzke M: Endogenous targets of RNA-directed DNA methylation and Pol IV in Arabidopsis. EMBO J 2006 25:2828-2836.

55. Erhard KF, Parkinson SE, Gross SM, Barbour J-ER, Lim JP, Hollick JB: Maize RNA polymerase IV defines transgenerational epigenetic variation. Plant Cell 2013, 25:808-819.

56. Mathieu O, Reinders J, Caikovski M, Smathajitt C, Paszkowski J: Transgenerational stability of the Arabidopsis epigenome is coordinated by CG methylation. Cell 2007, 130:851-862.

57. Stroud H, Do T, Du J, Zhong X, Feng S, Johnson L, Patel DJ, Jacobsen SE: Non-CG methylation patterns shape the epigenetic landscape in Arabidopsis. Nat Struct Mol Biol 2014, 21:64-72.

58. Lister R, O'Malley RC, Tonti-Filippini J, Gregory BD, Berry CC, Millar AH, Ecker JR: Highly integrated single-base resolution maps of the epigenome in Arabidopsis. Cell 2008, 133:523-536.

59. Li S, Vandivier LE, Tu B, Gao L, Won SY, Li S, Zheng B,

- Gregory BD, Chen X: Detection of Pol IV/RDR2-dependent transcripts at the genomic scale in Arabidopsis reveals features and regulation of siRNA biogenesis. Genome Res 2015, 25:235-245.

This publication identifies the Pol IV/RDR2 derived transcripts genome wide, as well as defines these regions as separate from those that are undergoing maintenance $\mathrm{CHH}$ methylation by CMT2.

60. Gent JI, Madzima TF, Bader R, Kent MR, Zhang X, Stam M, - McGinnis KM, Dawe RK: Accessible DNA and relative depletion of H3K9me2 at maize loci undergoing RNA-directed DNA methylation. Plant Cell 2014, 26:4903-4917.

This publication demonstrated that in the maize genome the RdDM pathway does not target the abundant heterochromatic blocks undergoing maintenance methylation, but rather TE regions that are adjacent to genes.

61. Blevins T, Pontvianne F, Cocklin R, Podicheti R, Chandrasekhara C, Yerneni S, Braun C, Lee B, Rusch D, Mockaitis $\mathrm{K}$ et al.: A two-step process for epigenetic inheritance in Arabidopsis. Mol Cell 2014, 54:30-42.

62. Saze H: Epigenetic memory transmission through mitosis and meiosis in plants. Semin Cell Dev Biol 2008, 19:527-536.

63. Becker C, Hagmann J, Müller J, Koenig D, Stegle O, Borgwardt K, Weigel D: Spontaneous epigenetic variation in the Arabidopsis thaliana methylome. Nature 2011, 480:245-249.

64. Daxinger L, Whitelaw E: Understanding transgenerational epigenetic inheritance via the gametes in mammals. Nat Rev Genet 2012, 13:153-162.

65. Baubec T, Finke A, Mittelsten Scheid O, Pecinka A: Meristemspecific expression of epigenetic regulators safeguards transposon silencing in Arabidopsis. EMBO Rep 2014, 15:446-452.

66. Martinez G, Slotkin RK: Developmental relaxation of transposable element silencing in plants: functional or byproduct? Curr Opin Plant Biol 2012, 15:496-502.

67. Schoft VK, Chumak N, Mosiolek M, Slusarz L, Komnenovic V, Brownfield L, Twell D, Kakutani T, Tamaru H: Induction of RNAdirected DNA methylation upon decondensation of constitutive heterochromatin. EMBO Rep 2009, 10:1015-1021.

68. Slotkin RK, Vaughn M, Borges F, Tanurdzic M, Becker JD, Feijó JA, Martienssen RA: Epigenetic reprogramming and small RNA silencing of transposable elements in pollen. Cell 2009 , 136:461-472.

69. Calarco JP, Borges F, Donoghue MTA, Van Ex F, Jullien PE, Lopes T, Gardner R, Berger F, Feijó JA, Becker JD et al.: Reprogramming of DNA methylation in pollen guides epigenetic inheritance via small RNA. Cell 2012, 151: 194-205. 
70. Pillot M, Baroux C, Vazquez MA, Autran D, Leblanc O, VielleCalzada J-P, Grossniklaus U, Grimanelli D: Embryo and endosperm inherit distinct chromatin and transcriptional states from the female gametes in Arabidopsis. Plant Cell 2010, 22:307-320.

71. Ibarra CA, Feng X, Schoft VK, Hsieh TF, Uzawa R, Rodrigues JA, Zemach A, Chumak N, Machlicova A, Nishimura T et al.: Active DNA demethylation in plant companion cells reinforces transposon methylation in gametes. Science 2012, 337: 1360-1364.

72. Gehring M, Bubb KL, Henikoff S: Extensive demethylation of repetitive elements during seed development underlies gene imprinting. Science 2009, 324:1447-1451.

73. Bourchis D, Voinnet O: A small-RNA perspective on gametogenesis fertilization, and early zygotic development. Science 2010, 330:617-622.

74. Brennecke J, Malone CD, Aravin AA, Sachidanandam R, Stark A, Hannon GJ: An epigenetic role for maternally inherited piRNAs in transposon silencing. Science 2008, 322:1387-1392.

75. $\mathrm{Li} \mathrm{H}$, Freeling M, Lisch D: Epigenetic reprogramming during vegetative phase change in maize. Proc Natl Acad Sci U $S A$ 2010, 107:22184-22189.

76. Mirouze M, Reinders J, Bucher E, Nishimura T, Schneeberger K, Ossowski S, Cao J, Weigel D, Paszkowski J, Mathieu O: Selective epigenetic control of retrotransposition in Arabidopsis. Nature 2009, 461:427-430.

77. Rudenko GN, Ono A, Walbot V: Initiation of silencing of maize MuDR/Mu transposable elements. Plant J 2003, 33:1013-1025.

78. Bennetzen JL, Wang $\mathrm{H}$ : The contributions of transposable elements to the structure, function, and evolution of plant genomes. Annu Rev Plant Biol 2014, 65:505-530.

79. Fu Y, Kawabe A, Etcheverry M, Ito T, Toyoda A, Fujiyama A, Colot V, Tarutani Y, Kakutani T: Mobilization of a plant transposon by expression of the transposon-encoded antisilencing factor. EMBO J 2013, 32:2407-2417.

80. Lisch D, Slotkin RK: Strategies for silencing and escape: the ancient struggle between transposable elements and their hosts. Int Rev Cell Mol Biol 2011, 292:119-152.

81. Joly-Lopez Z, Bureau TE: Diversity and evolution of transposable elements in Arabidopsis. Chromosome Res 2014 22:203-216.

82. McCue AD, Nuthikattu S, Slotkin RK: Genome-wide identification of genes regulated in trans by transposable element small interfering RNAs. RNA Biol 2013, 10:1379-1395.

83. Liu J, He $\mathrm{Y}$, Amasino $\mathrm{R}$, Chen $\mathrm{X}$ : siRNAs targeting an intronic transposon in the regulation of natural flowering behavior in Arabidopsis. Genes Dev 2004, 18:2873-2878.

84. Singh J, Freeling M, Lisch D: A position effect on the heritability of epigenetic silencing. PLOS Genet 2008, 4:e1000216.

85. Kabelitz T, Kappel C, Henneberger K, Benke E, Nöh C, Bäurle I: eQTL mapping of transposon silencing reveals a positiondependent stable escape from epigenetic silencing and transposition of AtMu1 in the Arabidopsis lineage. Plant Cell 2014, 26:3261-3271.

86. Zhu J, Kapoor A, Sridhar VV, Agius F, Zhu J-K: The DNA glycosylase/lyase ROS1 functions in pruning DNA methylation patterns in Arabidopsis. Curr Biol 2007, 17:54-59.

87. Saze H, Shiraishi A, Miura A, Kakutani T: Control of genic DNA methylation by a jmiC domain-containing protein in Arabidopsis thaliana. Science 2008, 319:462-465.

88. Rigal M, Kevei Z, Pélissier T, Mathieu O: DNA methylation in an intron of the IBM1 histone demethylase gene stabilizes chromatin modification patterns. EMBO J 2012, 31:2981-2993.

89. Panda K, Slotkin RK: Proposed mechanism for the initiation of transposable element silencing by the RDR6-directed DNA methylation pathway. Plant Signal Behav 2013:8. 